\title{
Analyzing Active Interactive Genetic Algorithms using Visual Analytics
}

\author{
Xavier Llorà \\ Automated Learning Group \\ NCSA \\ University of Illinois \\ Urbana, IL 61801, USA \\ xllora@illigal.ge.uiuc.edu
}

\author{
Kumara Sastry \\ IlliGAL \\ Department of IESE \\ University of Illinois \\ Urbana, IL 61801, USA \\ kumara@illigal.ge.uiuc.edu
}

\author{
Francesc Alías \\ Com. and Signal Theory \\ Arquitectura La Salle \\ Universitat Ramon Llull \\ 08022 Barcelona, Spain \\ falias@salleurl.edu
}

\begin{abstract}
This paper builds introduces visual-analytic techniques to aggregate, summarize, and visualize the information generated during interactive evolutionary processes. Special visualizations of the user-provided partial ordering of solutions, the synthetic fitness surrogates induced, and the model of user preferences were prepared. The proposed visualanalytic techniques point out potential pitfalls, strengths, and possible improvements in a non-trivial case study where the hierarchical tournament selection scheme of an active interactive genetic algorithm is replaced by an incremental selection scheme. Visual analytics provided an intuitive reasoning environment that unveiled important properties that greatly affect the performance of active interactive genetic algorithms that could not have been easily reveled otherwise.
\end{abstract}

\section{Categories \& Subject Descriptors}

I.2.6.f [Artificial Intelligence]: Knowledge adquisition.

\section{General Terms}

Algorithms, Design, Theory, Experimentation.

\section{Keywords}

Interactive genetic algorithms, active learning, visual analytics, model learning.

\section{INTRODUCTION}

Active interactive genetic algorithms (aiGAs) [3] mine models of users preferences during interactive sessions for problem solving. It builds on the partial ordering of solutions provided by the user evaluations. Traditional interactive genetic algorithms (iGA) [1] require the user to provide a large number of evaluations to achieve good quality solutions. One of the main contributions of aiGA is the use of mined models of user preferences to generate educated guesses - promising solutions. Presenting educated guesses to the user helps reducing the fatigue - the user can obtain high-quality solutions faster - and the frustrationgenerating high-quality solutions allows aiGA to avoid the

Copyright is held by the author/owner(s).

GECCO'06, July 8-12, 2006, Seattle, Washington, USA.

ACM 1-59593-186-4/06/0007. repetitive display of poor solutions that may discourage the user.

The fusion of human and computer capabilities in aiGA provides large amounts of information not used during the interactive sessions. This paper introduces visual analytics techniques to aggregate, summarize, and visualize the information generated during the interactive process. Special visualizations of the user-provided partial ordering of solutions, the synthetic fitness surrogates induced, and the model of user preferences were prepared. The visualizations allow an observer to evaluate at a glance the progress of the interactive process so far through a non-interfering looking glass. The visual-analytic tools have been successfully used to analyze the behavior of users during an interactive weight-tuning of a cost function involved with corupusbased text-to-speech synthesis [2]. Additionally, the visualanalytic techniques point out potential pitfalls, strengths, and possible improvements of the active interactive genetic algorithms. In this paper, we demonstrate the effectiveness of the visualization techniques with the help of a non-trivial case study.

\section{VISUAL ANALYTICS}

Visual analytics is the science of analytical reasoning facilitated by interactive visual interfaces [6]. In order to be effective, visualizations need to satisfy some principles. A detailed explanation is beyond the scope of this paper and can be found elsewhere [6]. We would briefly describe the three principles proposed by Norman in 1993 [4]. The appropriateness principle says that a given visualization should provide neither more nor less information than the one at hand. The naturalness principle postulates that a visual representations should closely match the information being represented. And the last principle, the matching principle, says that representations of information are most effective when they match the task to be performed by the user.

However, visual analytics does not only involve visualizations, it also relies on the interaction between the user and the analytic tools. In the aiGA response time up to a minute are acceptable - as already stated by other researches [6] beyond which it will only increase the fatigue and frustration of the users. Usual interaction modes for visual analytics covers filtering, navigation, and discourse interpretation. Interactive genetic algorithms can be regarded as computational embodiments to navigate across a given search space under the human guidance [5]. Moreover, aiGA learn from that guided navigation, thus, visual analytics techniques are 
a perfect aid to reason about the dynamics of the interactive process.

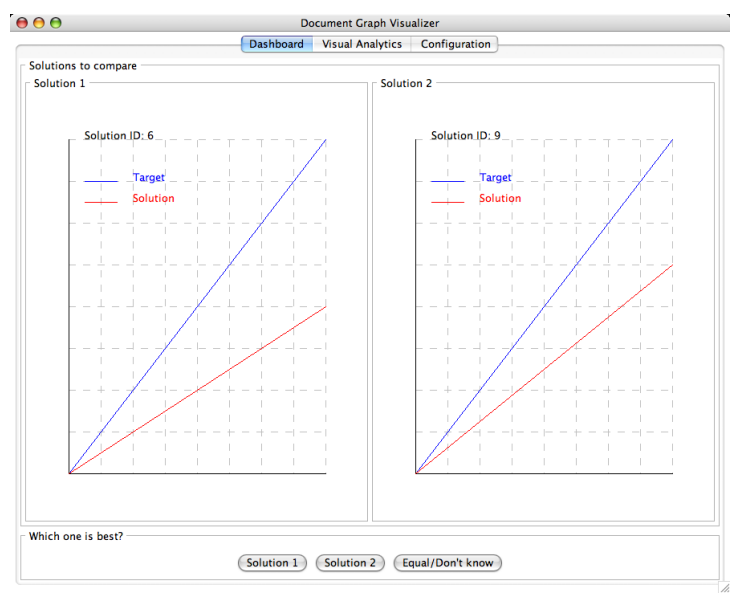

(a) Dashboard

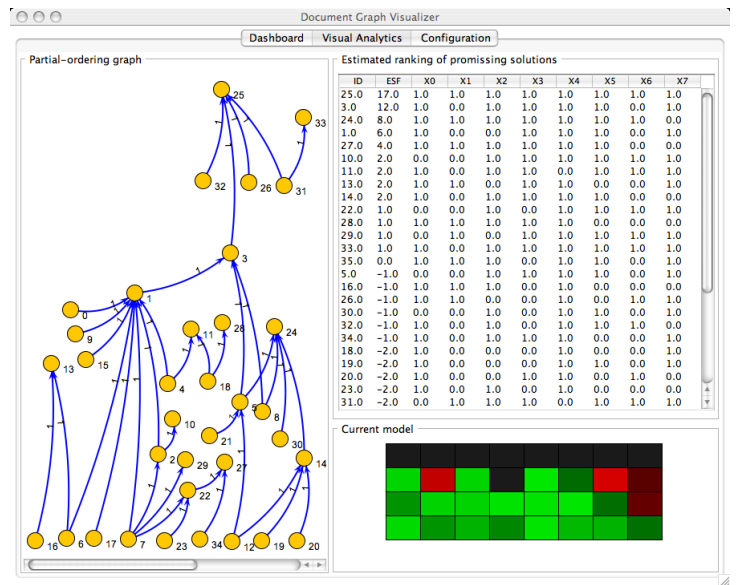

(b) Visual analytics

Figure 1: The user interacts on a simple dashboard. The anlysis of the active interactive GA run is displayed on a specificly dessigned visual analytics area.

\section{VISUAL ANALYSIS OF AIGA BEHAVIOR}

Visual analytics can be a powerful tool to analyze aiGA behavior. The visual analytics tools cover the three main properties introduced in the previous section: (1) user evaluation inconsistencies via the partial-ordering graph, (2) the misalignment of the estimated fitness and the synthetic surrogate model, and (3) the dynamics of the learned probabilistic models of the user preferences.

Three visualization tools were developed taking into acount the principles introduced in the previous section. Figure 1(b) presents the visual analytics techniques to aggregate, summarize, and visualize the information generated by aiGA. These tools were used in a case study where where we modified the selection phase of the aiGA to removed the hierarchical tournament scheme proposed by Llorà et al [3] replacing it by an incremental construction of the graph, dropping a comparison if considered equal. Dropping the comparison removes the need to normalize the resulting partial-ordering graph [3] and minimize the differences with the original method. However, using the visual analystics techniques introduced, we identified that dropping a compasion - instead of keeping it as the original hierarchical tournament does - eventually degraded the performance of the overall aiGA, requiring similar number of evaluations as the simple GA. Visual analytics tools also showed that the way the partial ordering graph is created has a significant impact on the quality of the surrogate fitnesses build. We are currently focusing on better understanding the relation between the partial ordering graph and the surrogate fitness to produce better tournament schemes that do not disrupt the learning of the surrogate model.

\section{ACKNOWLEDGMENTS}

This work was sponsored by the AFOSR (F49620-03-10129) and NSF (ISS-02-09199).

\section{ADDITIONAL AUTHORS}

Additional authors: David E. Goldberg (IlliGAL, Department of IESE, University of Illinois, Urbana, IL 61801, USA, email: deg@uiuc.edu) and Michael Welge (Automated Learning Group, NCSA, University of Illinois,Urbana, IL 61801, USA, email:welge@ncsa.uiuc.edu)

\section{REFERENCES}

[1] C. Caldwell and V. S. Johnston. Tracking a criminal suspect through face-space with a genetic algorithm. Proceedings of the Fourth International Conference on Genetic Algorithms, pages 416-421, 1991.

[2] X. Llorà, F. Alías, L. Formiga, K. Sastry, and D. E. Goldberg. Evaluation consistency in iGAs: User contradictions as cycles in partial-ordering graphs. In IEEE International Conference on Acoustics, Speech, and Signal Processing (ICASSP 2006), page in press, 2006. Also as IlliGAL TR No. 2005022.

[3] X. Llorà, K. Sastry, D. E. Goldberg, A. Gupta, and L. Lakshmi. Combating user fatigue in iGAs: partial ordering, support vector machines, and synthetic fitness. In GECCO 2005: Proceedings of the 2005 conference on Genetic and evolutionary computation, volume 2, pages 1363-1370, Washington DC, USA, 25-29 June 2005. ACM Press.

[4] D. E. Norman. Things That Make Us Smart: Defending Human Attributes in the Age of the Machine. Perseus Books, New York, 1993.

[5] H. Takagi. Interactive evolutionary computation: Fusion of the capabilities of EC optimization and human evaluation. Proceedings of the IEEE, 89(9):1275-1296, 2001.

[6] J. J. Thomas and K. A. Cook. Illuminating the Path: The Research and Development Agenda for Visual Analytics. IEEE press, 2005. 